and Hand), STEF (Simple Test for Evaluating Hand Function), and Purdue Pegboard.

Methods: We developed the following equipment: Type $U$ to imitate extension limitation of metacarpophalangeal (MCP) joints seen in ulnar deviation; Type B which imitates flexion deformity of the distal interphalangeal (DIP) joints by seen in boutonniere deformity; and Type $S$ which imitates flexion limitation of proximal interphalangeal (PIP) and interphalangeal (IP) joints by reversing the upper and lower ends of the Oval-8 Finger Splint (Fukui Co. Ltd, Japan). Types U and S were fitted on HV (index to pinky). RSE was evaluated using DASH, STEF and Purdue Pegboard in hand-finger function evaluation. Twenty-four RA patients with handfinger deformation and Forty-one HV were included in this study to evaluate the equipment.

Results: Mean \pm SD ages for RA patients was $67.4 \pm 8.0$ years (95.8\% female) and $38.2 \pm 17.7$ for HV (63.4\% female), respectively. Total hand-finger deformities for RA patients were 23 hands for ulnar deviation, 66 fingers for swan-neck deformity, and 33 fingers for boutonniere deformity. Randomization for RA patients was as follows: 13 DASH, 5 (10 hands) STEF, and 6 Purdue Pegboard. $10 \mathrm{HV}$ were assigned to DASH, 10 to Purdue Pegboard, and 14 (28 hands) to STEF. HV were evaluated with RSE and without RSE. For DASH, STEF, and Purdue Pegboard, RA patients showed significant functional loss compared to HV. Significant function loss in RA patients was also observed with the RSE. However, no differences were seen between the RA group and the HV with RSE group (figure 1).

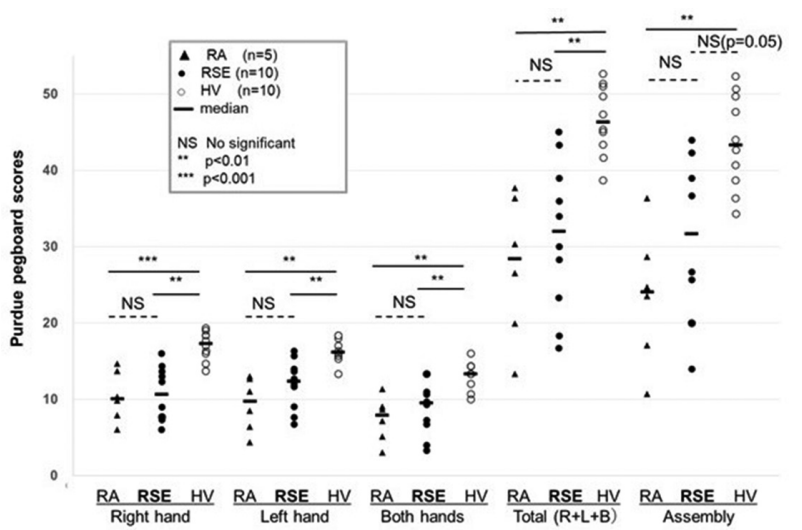

Abstract THU0734HPR - Figure 1 Purdue. pegboard scores of RA, RSE and HV groups

Conclusions: We developed the RSE, which allows for one to experience the decrease in function with RA hand-finger deformity. Our study showed that RSE use can indeed allow this experience. By using RSE, health care workers, patient caretakers and early RA patients can experience joint limitation of RA for educational purposes, personalised rehab programs, and development of self-help tools.

Disclosure of Interest: None declared

DOI: 10.1136/annrheumdis-2018-eular.3416

\section{THU0735-HPR HOW RHEUMATOLOGY SPECIALIST NURSE DETECTS SMOKING HABIT IN RHEUMATOID CHRONIC INFLAMMATORY DISEASE PACIENTS}

M. Serra Miralles ${ }^{1}$, F. Abella ${ }^{2}$, N. Montalà ${ }^{1}$, M. Mateu ${ }^{1}$, A. Palau ${ }^{1}$, M. Conde ${ }^{1}$, L. Roselló ${ }^{1}$ 'Rheumatology, ${ }^{2}$ Psychology, Hospital Santa Maria, Lleida, Spain

Background: Inflammation plays a significant role in the development of atherosclerosis and cardiovascular disease (CVD). Patients with chronic inflammatory disease (CID) have an increased risk of CVD. Smoking is one of the main risk factors for CVD and a predictor of poor response to treatment and poor prognosis.

Objectives: We aimed to describe the role on a rheumatology specialist nurse in the detection of smoking habit and dependence to nicotine in CID patients before the start of an interventionism plan to avoid it in CID patients.

Methods: All CID patients attended in a nurse rheumatologist unit, during one month, were asked for smoking habit. We determined the characteristics, onset of smoking and number of cigarettes per day. The Fagerström test was used to establish the nicotine dependence (ND) (score >5) and the Richmond test for predicting abstention following intervention to stop smoking (score $>5$ ). Exhaled $\mathrm{CO}$ level Coximetry was also recorded. We planned an interventionism consenting in health advice provided by a nurse and the derivation to a smoke unit.

Results: 22 patients were identified. $12(50.0 \%)$ were female and mean age was 46.0 (SD 10.5). 14 (63.6\%) suffered Rheumatoid Arthritis, 6 (27.3\%) Ankylosing Spondylitis and $2(9.1 \%)$ Psoriatic Arthritis. 5 (22.7\%) subjects were under biological treatment. Mean number of cigarettes smoked per day and smoking years were 16.0 (SD 8.9) cigarettes and 27.2 (SD 11.7) years respectively. Up to 5 out of $22(22.7 \%)$ subjects had high ND. Patients with ND had higher exhaled CO levels than non ND subjects (24.6 [SD 6.0] vs. 17.9 [SD 12.2] p-value 0.160). We observed no differences in age, sex, rheumatoid diagnosis, years smoking or cigarettes per day between both groups. Active treatment with biological drugs was significantly associated with ND (60.0 vs. $14.3 \%$, p-value 0.046$)$. One out of five patients with ND had good abstention prediction according to Richmond test Conclusions: Nearly one out of five smoking CID subjects had severe ND. Treatment with biological drugs was related to ND. Interestingly, most of the ND subjects had good prediction for smoking cessation. Therefore, the development of a nurse program to detect smoking and our interventionism plan to avoid smoking habit are of enormous interest.

\section{REFERENCES}

[1] Oliver S, Leary A. The value of the nurse specialists' role: Pandora initial findings. Musculoskeletal Care 2010;8:175-7.

[2] Soderlin MK, Petersson IF, Geborek P. The effect of smoking on response and drug survival in rheumatoid arthritis patients treated with their first antiTNF drug. Scand J Rheumatol 2012;41:1-9.

[3] Chang K, Yang SM, Kim SH, Han KH, Park SJ, Shin JI. Smoking and rheumatoid arthritis. Int J Mol Sci 2014;15:22279-95.

[4] Roelsgaard IK, Thomsen T, Ostergaard M, et al. The effect of an intensive smoking cessation intervention on disease activity in patients with rheumatoid arthritis: study protocol for a randomised controlled trial. Trials 2017;18:570

Disclosure of Interest: None declared

DOI: 10.1136/annrheumdis-2018-eular.4105

\section{THU0736-HPR THE EFFECTIVENESS OF SHORT TRAINING IN PSYCHOLOGICAL SUPPORT FOR NURSES IN RHEUMATIC CARE}

M. Fusama ${ }^{1}$, H. Nakahara ${ }^{2}$, K. Higashi ${ }^{1}$, M. Mori ${ }^{3}$, M. Mabuchi ${ }^{4}$, Y. Sakai ${ }^{5}$, T. Motonaga ${ }^{6}$, T. Takeuchi ${ }^{7}{ }^{1}$ Division of Nursing; ${ }^{2}$ NTT West Osaka Hospital, Osaka; ${ }^{3}$ Jikei University School of Medicine; ${ }^{4}$ Tokyo University of Agriculture and Technology, ${ }^{5}$ Atomi University; ${ }^{6}$ Teikyo University, ${ }^{7}$ Department of Internal Medicine, Keio University School of Medicine, Tokyo, Japan

Background: Patients with rheumatoid arthritis (RA) often suffer from psychological concerns. ${ }^{1}$ There is a lack of psychology specialists in many hospitals and clinics. According to EULAR, nurses should undertake a significant portion of providing psychological support to patients. ${ }^{2}$ However, in Japan nurses involved in clinical practice often do not have sufficient time and opportunity to receive adequate education on psychological support.

Objectives: The aim of this study is to evaluate nurses' understanding of basic psychological concepts and the efficacy of a short psychology workshop for nurses in RA clinical care.

Methods: A clinical psychotherapist provided a lecture on psychological support once for a period of $30 \mathrm{~min}$, followed by 1 hour of role-play groupwork. The lecture focused on basic psychological support including concepts of "listening", "acceptance", "empathy", "open question", "closed question" and "patient-centred perspective". Group work focused on applying the aforementioned concepts. Nurses were asked before and after workshop regarding their understanding and opinions on the necessity and feasibility of psychological support, as well as their motivation for implementation. Nurses' opinions were evaluated on a 1-7 rating scale (1=not at all, 7=full). Client Satisfaction Questionnaire-8 (CSQ-8J) was utilised to assess satisfaction with the workshop. Data analyses were performed with Wilcoxon signed rank test.

Results: 53 nurses (M/F: 1/52) participated in this study. The average of ages clinical experience and clinical experience in RA care were 45.5 years old, 21.0 and 7.9 years, respectively. Nurses' opinions regarding the necessity and feasibility of psychological support in general, as well as their motivation for providing psychological support statistically significantly increased post-workshop (necessity: $p=0.0052$, feasibility: $p<0.0001$, motivation: $p=0.0033$ )

Nurses' answers regarding their current application of these concepts in clinica practice were as follows: mean $\pm S D$; listening: $5.15 \pm 1.20$, empathy: $5.06 \pm 1.18$ acceptance: $4.71 \pm 1.18$, open question: $4.80 \pm 1.31$, closed question: $4.49 \pm 1.47$. The understanding of these concepts was statistically significantly higher after the workshop ( $p<0.0001$ for each concept). Moreover, the feasibility of all the above concepts was rated as statistically significantly higher post-workshop. $(p<0.001$ for each concept). The necessity of adopting a patient centred perspective was also judged as statistically significantly higher post-workshop $(p<0.001)$. Based on CSQ-8J, satisfaction with the workshop was overall high.

Conclusions: This is the first report evaluating the effectiveness of brief psychological workshops for nurses in rheumatic care in Japan. This preliminary study 